Pacific

Journal of

Mathematics

TORUS ACTIONS AND TENSOR PRODUCTS OF INTERSECTION COHOMOLOGY

ASILATA BAPAT

Volume $276 \quad$ No. 1

July 2015 


\title{
TORUS ACTIONS AND TENSOR PRODUCTS OF INTERSECTION COHOMOLOGY
}

\author{
ASILATA BAPAT
}

\begin{abstract}
Given certain intersection cohomology sheaves on a projective variety with a torus action, we relate the cohomology groups of their tensor product to the cohomology groups of the individual sheaves. We also prove a similar result in the case of equivariant cohomology.
\end{abstract}

\section{Introduction}

Let $X$ be a smooth complex projective variety together with an action of a complex algebraic torus $T$ with isolated fixed points. We fix a regular algebraic one-parameter subgroup $\lambda: \mathbb{C}^{*} \rightarrow T$, which means that the set of $\lambda$-fixed points on $X$ equals the set of $T$-fixed points on $X$ (denoted $X^{T}$ ). Consider the Białynicki-Birula decomposition [1973] of $X$ : for each $w \in X^{T}$ define the plus and minus cells to be respectively

$$
\begin{aligned}
U_{w}=U_{w}^{+} & =\left\{x \in X \mid \lim _{t \rightarrow 0} \lambda(t) \cdot x=w\right\}, \quad t \in \mathbb{C}^{*}, \text { and } \\
U_{w}^{-} & =\left\{x \in X \mid \lim _{t \rightarrow \infty} \lambda(t) \cdot x=w\right\}, \quad t \in \mathbb{C}^{*} .
\end{aligned}
$$

Each plus or minus cell is a $\lambda$-stable affine space, and hence the decompositions $X=\bigsqcup_{w \in X^{T}} U_{w}$ and $X=\bigsqcup_{w \in X^{T}} U_{w}^{-}$are cell decompositions. For the purposes of this paper, we make the following additional assumptions on the $T$-action on $X$.

Assumption 1.1. The cell decompositions $X=\bigsqcup_{w \in X^{T}} U_{w}$ and $X=\bigsqcup_{w \in X^{T}} U_{w}^{-}$ are algebraic stratifications of $X$. In particular, the closure of every plus cell is a union of plus cells, and analogously for minus cells.

Assumption 1.2. For each $w \in X^{T}$, there is a one-parameter subgroup $\lambda_{w}: \mathbb{C}^{*} \rightarrow T$ and a neighborhood $V_{w}$ of $w$ such that $\lim _{t \rightarrow 0} \lambda_{w}(t) \cdot v=w$ for every $v \in V_{w}$ and $t \in \mathbb{C}^{*}$.

In this paper, we use the words sheaf and complex of sheaves interchangeably to mean an object in $D_{c, \mathrm{BB}}^{b}(X, \mathbb{C})$, the bounded derived category of sheaves of $\mathbb{C}$-vector spaces on $X$ that are constructible with respect to the Białynicki-Birula

MSC2010: 14F05, 14F43, 14L30, 55N33.

Keywords: intersection cohomology, torus action. 
stratification. (Here we make use of Assumption 1.1.) Moreover all functors are derived, so for ease of notation we omit the decorations $\mathrm{R}$ and $\mathrm{L}$.

For each $w \in X^{T}$, let $\mathrm{IC}_{w}$ denote the intersection cohomology sheaf on the closure of the cell $U_{w}$, extended by zero to all of $X$. The main theorem of the paper describes the cohomology of the tensor products of a collection of $\mathrm{IC}_{w}$, in terms of the tensor products of the cohomologies of the individual $\mathrm{IC}_{w}$.

Main result. Let $\Delta: X \rightarrow X^{m}$ be the diagonal embedding. Consider any sheaves $\mathscr{F}_{1}, \ldots, \mathscr{F}_{m}$ in $D_{c, \mathrm{BB}}^{b}(X, \mathbb{C})$. Then their (derived) tensor product is also a sheaf in $D_{c, \mathrm{BB}}^{b}(X, \mathbb{C})$, and will be denoted by $\mathscr{F}_{1} \otimes \cdots \otimes \mathscr{F}_{m}$. Recall that

$$
\mathscr{F}_{1} \otimes \cdots \otimes \mathscr{F}_{m}=\Delta^{-1}\left(\mathscr{F}_{1} \otimes \cdots \otimes \mathscr{F}_{m}\right) .
$$

For any sheaf $\mathscr{F}$, its cohomology $H^{\bullet}(\mathscr{F})=H^{\bullet}(X, \mathscr{F})$ is a graded vector space. There is a natural cup product $\cup: H^{\bullet}\left(\mathscr{F}_{1}\right) \otimes \cdots \otimes H^{\bullet}\left(\mathscr{F}_{m}\right) \rightarrow H^{\bullet}\left(\mathscr{F}_{1} \otimes \cdots \otimes \mathscr{F}_{m}\right)$, defined on page 22 .

Let $\mathbb{C}$ denote the constant sheaf on $X$. For any sheaf $\mathscr{F}$, its cohomology $H^{\bullet}(\mathscr{F})$ is naturally a (graded) left and right module over the (graded) ring $H(X)=H^{\bullet}(X, \underline{\mathbb{C}})$, as follows:

$$
\begin{aligned}
& \cup: H(X) \otimes H^{\bullet}(\mathscr{F}) \rightarrow H^{\bullet}(\underline{\mathbb{C}} \otimes \mathscr{F}) \stackrel{\cong}{\rightarrow} H^{\bullet}(\mathscr{F}), \\
& \cup: H^{\bullet}(\mathscr{F}) \otimes H(X) \rightarrow H^{\bullet}(\mathscr{F} \otimes \underline{C}) \stackrel{\cong}{\rightarrow} H^{\bullet}(\mathscr{F}) .
\end{aligned}
$$

Moreover, the cup product descends to a morphism

$$
H^{\bullet}\left(\mathscr{F}_{1}\right) \underset{H(X)}{\otimes} \cdots \underset{H(X)}{\otimes} H^{\bullet}\left(\mathscr{F}_{m}\right) \rightarrow H^{\bullet}\left(\mathscr{F}_{1} \otimes \cdots \otimes \mathscr{F}_{m}\right) .
$$

Theorem 1.3. Let $\left(p_{1}, \ldots, p_{m}\right)$ be an $m$-tuple of $T$-fixed points of $X$, and suppose that Assumptions 1.1 and 1.2 hold. Then the cup product map

$$
H^{\bullet}\left(\mathrm{IC}_{p_{1}}\right) \underset{H(X)}{\otimes} \cdots \underset{H(X)}{\otimes} H^{\bullet}\left(\mathrm{IC}_{p_{m}}\right) \rightarrow H^{\bullet}\left(\mathrm{IC}_{p_{1}} \otimes \cdots \otimes \mathrm{IC}_{p_{m}}\right)
$$

is an isomorphism.

As $X$ is a $T$-space, each IC sheaf $\mathrm{IC}_{p_{j}}$ carries a canonical $T$-equivariant structure, and so does the tensor product $\mathrm{IC}_{p_{1}} \otimes \cdots \otimes \mathrm{IC}_{p_{m}}$. Let $H_{T}(X)=H_{T}^{\bullet}(X, \mathbb{C})$ be the $T$-equivariant cohomology of $X$. For any $T$-equivariant sheaf $\mathscr{F}$ on $X$, its $T$-equivariant cohomology $H_{T}^{\bullet}(\mathscr{F})=H_{T}^{\bullet}(X, \mathscr{F})$ is a graded $H_{T}(X)$-module. As before, there is a cup product map for $T$-equivariant cohomology, which factors through $H_{T}(X)$.

Theorem 1.4. Under Assumptions 1.1 and 1.2, the cup product map

is an isomorphism.

$$
H_{T}^{\bullet}\left(\mathrm{IC}_{p_{1}}\right) \underset{H_{T}(X)}{\otimes} \cdots \underset{H_{T}(X)}{\otimes} H_{T}^{\bullet}\left(\mathrm{IC}_{p_{m}}\right) \rightarrow H_{T}^{\bullet}\left(\mathrm{IC}_{p_{1}} \otimes \cdots \otimes \mathrm{IC}_{p_{m}}\right)
$$


Remark 1.5. Even though our results are stated using IC sheaves, it is possible that they generalize to parity sheaves (defined and discussed by Juteau, Mautner, and Williamson in [Juteau et al. 2014]). Our results and proof methods are similar to the main theorem from [Ginzburg 1991]. Achar and Rider [2014, Theorem 4.1] prove a version of Ginzburg's theorem for parity sheaves on generalized flag varieties of a Kac-Moody group. Similar generalizations may work in our case as well.

\section{Setup}

The Biatynicki-Birula stratification. One can find (see, e.g., [Sumihiro 1974] or [Kambayashi 1966]) a $T$-equivariant projective embedding of $X$ into some $\mathbb{P}^{N}$, such that the action of $T$ on $\mathbb{P}^{N}$ is linear. Consider the following standard Morse-Bott function on $\mathbb{P}^{N}$ :

$$
\left[z_{0}: \cdots: z_{N}\right] \mapsto \frac{\sum_{i=0}^{N} c_{i}\left|z_{i}\right|^{2}}{\sum_{i=0}^{N}\left|z_{i}\right|^{2}},
$$

where $c_{i}$ are the weights of the $\lambda$-action on $\mathbb{P}^{N}$. The critical sets of this function are precisely the $T$-fixed points on $\mathbb{P}^{N}$. The Morse-Bott cells of this function are locally closed algebraic subvarieties of $\mathbb{P}^{N}$. Since $X$ has isolated $T$-fixed points, one can show that the composition $f: X \rightarrow \mathbb{P}^{N} \rightarrow \mathbb{R}$ is a Morse function with critical set $X^{T}$ (see, e.g., [Audin 2004]). Each cell of the Morse decomposition under $f$ is a preimage of a Morse-Bott cell of $\mathbb{P}^{N}$. Hence it is a locally closed algebraic subvariety of $X$. Moreover, each cell of the Morse decomposition is known to be a union of Białynicki-Birula plus cells. A discussion of this may also be found in [Chriss and Ginzburg 1997, Section 2.4].

The collection of fixed points of the $\lambda$-action carries a partial order, where $v<w$ if $U_{v} \subset \overline{U_{w}}$. By the previous discussion, we see that $v<w$ if and only if $f(v)<f(w)$. Fix a weakly increasing enumeration $\{0,1, \ldots, N\}$ of the points of $X^{T}$ (sometimes denoted $\left\{w_{0}, \ldots, w_{N}\right\}$ ), and set $X_{n}=\bigcup_{i \leq n} U_{i}$. Since the closure of every plus cell is a union of plus cells, it follows from the previous discussion that each $X_{n}$ is a closed subvariety of $X$.

Similarly, set $X_{n}^{-}=\bigcup_{i \geq n} U_{i}^{-}$. By using the Morse function $(-f)$ instead of $f$, we see that each $X_{n}^{-}$is a closed subvariety of $X$. Hence we obtain two increasing filtrations of $X$ by closed subvarieties: $X_{0} \subset \cdots \subset X_{N}=X$ and $X_{N}^{-} \subset \cdots \subset X_{0}^{-}=X$.

We have the following inclusions:

$$
X_{n} \stackrel{i_{n}}{\hookrightarrow} X, \quad X_{n-1} \stackrel{v}{\hookrightarrow} X_{n} \stackrel{u}{\longleftrightarrow} U_{n} .
$$

For any point $p \in X_{n}^{-}$, we have $f\left(w_{n}\right) \leq f(p)$, with equality only if $p \in X^{T}$. For any point $p \in X_{n}$, we have $f(p) \leq f\left(w_{n}\right)$, with equality only if $p \in X^{T}$. Hence if $p \in X_{n}^{-} \cap X_{n}$, then $f(p)=f\left(w_{n}\right)$, and $p \in X^{T}$. But $X_{n}^{-} \cap X_{n} \cap X^{T}=\left\{w_{n}\right\}$, and 
it follows that $p=w_{n}$. Hence for every $n$, the subvarieties $X_{n}^{-}$and $X_{n}$ intersect transversally in the single point $w_{n}$.

Let $c_{n} \in H^{\bullet}(X)$ be the Poincaré dual to the homology class of $X_{n}^{-}$. As a vector space, $H^{\bullet}(X)$ is generated by the collection $\left\{c_{n}\right\}$. Finally, fix an $m$-tuple $\left(p_{1}, \ldots, p_{m}\right)$ of $T$-fixed points of $X$, and set $L_{j, n}=i_{n}^{-1} \mathrm{IC}_{p_{j}}$ for each $j$ and $n$.

The cup product in cohomology. Let $\pi: X \rightarrow$ pt be the unique morphism to a point. For any sheaf $\mathscr{F}$ on $X$, its cohomology $H^{\bullet}(\mathscr{F})$ is a graded vector space, and may be thought of as $\pi_{*} \mathscr{F}$. We use this to define the cup product map.

Recall that the functors $\left(\pi^{-1}, \pi_{*}\right)$ form an adjoint pair, which has a counit $\pi^{-1} \circ \pi_{*} \rightarrow$ id. Let $\mathscr{F}_{1}, \ldots, \mathscr{F}_{m}$ be sheaves on $X$. Tensoring the counit maps together, we have a map

$$
\pi^{-1} \circ \pi_{*}\left(\mathscr{F}_{1}\right) \otimes \cdots \otimes \pi^{-1} \circ \pi_{*}\left(\mathscr{F}_{m}\right) \rightarrow \mathscr{F}_{1} \otimes \cdots \otimes \mathscr{F}_{m}
$$

The left hand side is canonically isomorphic to $\pi^{-1}\left(\pi_{*} \mathscr{F}_{1} \otimes \cdots \otimes \pi_{*} \mathscr{F}_{m}\right)$. Using the $\left(\pi^{-1}, \pi_{*}\right)$ adjunction once more, we obtain the cup product:

$$
\cup: \pi_{*} \mathscr{F}_{1} \otimes \cdots \otimes \pi_{*} \mathscr{F}_{m} \rightarrow \pi_{*}\left(\mathscr{F}_{1} \otimes \cdots \otimes \mathscr{F}_{m}\right)
$$

The cup product gives each $H^{\bullet}\left(\mathscr{F}_{i}\right)$ the structure of a left and right module over $H(X)$. This module structure induces the following map, also called the cup product:

$$
H^{\bullet}\left(\mathscr{F}_{1}\right) \underset{H(X)}{\otimes} \cdots \underset{H(X)}{\otimes} H^{\bullet}\left(\mathscr{F}_{m}\right) \rightarrow H^{\bullet}\left(\mathscr{F}_{1} \otimes \cdots \otimes \mathscr{F}_{m}\right) .
$$

Proposition 2.1. For every $n$, the cup product map

$$
H^{\bullet}\left(L_{1, n}\right) \underset{H(X)}{\otimes} \cdots \underset{H(X)}{\otimes} H^{\bullet}\left(L_{m, n}\right) \rightarrow H^{\bullet}\left(L_{1, n} \otimes \cdots \otimes L_{m, n}\right)
$$

is an isomorphism.

When $X_{n}=X$, we have $L_{j, n}=\mathrm{IC}_{p_{j}}$ for each $j$. Hence Theorem 1.3 follows from this proposition, and we now focus on proving the proposition.

\section{Proof of the isomorphism}

We prove Proposition 2.1 by induction on the $n$th filtered piece of $X_{0} \subset \cdots \subset X_{N}$. In the base case of $n=0$, the space $X_{0}$ is zero-dimensional. Hence each sheaf $L_{j, 0}$ is isomorphic to its cohomology. In this case the cup product map (2-1) reduces to the identity map, which is an isomorphism. 
Now we prove the induction step on the filtered piece $X_{n}$. We mainly use the following distinguished triangles:

$$
\begin{aligned}
u_{!} u^{-1} L_{j, n} & \rightarrow L_{j, n} \rightarrow v_{*} v^{-1} L_{j, n}, \\
v_{!} v^{!} L_{j, n} & \rightarrow L_{j, n} \rightarrow u_{*} u^{-1} L_{j, n} .
\end{aligned}
$$

After taking cohomology, each of the above distinguished triangles produces a long exact sequence. In our case, all connecting homomorphisms of these long exact sequences vanish (see, e.g., [Soergel 1990, Lemma 20] and [Ginzburg 1991, Proposition 3.2]).

For brevity, we will use the following notation through the remainder of the paper.

$$
\begin{aligned}
M_{m, n} & =L_{2, n} \otimes \cdots \otimes L_{m, n}, \\
A_{m, n} & =H^{\bullet}\left(L_{2, n}\right) \underset{H(X)}{\otimes} \cdots \underset{H(X)}{\otimes} H^{\bullet}\left(L_{m, n}\right), \\
B_{m, n} & =H^{\bullet}\left(u_{*} u^{-1} L_{2, n}\right) \underset{H(X)}{\otimes} \cdots \underset{H(X)}{\otimes} H^{\bullet}\left(u_{*} u^{-1} L_{m, n}\right) .
\end{aligned}
$$

The following two lemmas prove the proposition on the open part $U_{n}$ in $X_{n}$.

Lemma 3.1. Let $\mathscr{F}$ and $\varphi$ be any complexes of sheaves on $U_{n}$ with locally constant cohomology sheaves. Then the cup product map

$$
\cup: H^{\bullet}\left(u_{!} \mathscr{F}\right) \otimes H^{\bullet}\left(u_{*} \mathscr{G}\right) \rightarrow H^{\bullet}\left(u_{!} \mathscr{F} \otimes u_{*} \mathscr{G}\right)
$$

is an isomorphism. Since $\cup$ factors through the surjection

$$
H^{\bullet}\left(u_{!} \mathscr{F}\right) \otimes H^{\bullet}\left(u_{*} \mathscr{G}\right) \rightarrow H^{\bullet}\left(u_{!} \mathscr{F}\right) \underset{H(X)}{\otimes} H^{\bullet}\left(u_{*} \mathscr{G}\right),
$$

the induced cup product

$$
\cup: H^{\bullet}\left(u_{!} \mathscr{F}\right) \underset{H(X)}{\otimes} H^{\bullet}\left(u_{*} \mathscr{G}\right) \rightarrow H^{\bullet}\left(u_{!} \mathscr{F} \otimes u_{*} \mathscr{G}\right)
$$

is also an isomorphism.

Proof. Consider the following commutative diagram, where $\pi$ is the projection to a point.

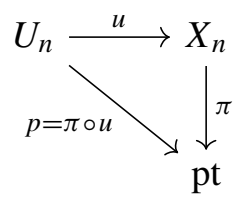


Recall that if $A$ and $B$ are any two complexes on $X$, then the cup product is induced by adjunction from the natural map

$$
\pi^{-1}\left(\pi_{*} A \otimes \pi_{*} B\right) \cong \pi^{-1} \pi_{*} A \otimes \pi^{-1} \pi_{*} B \rightarrow A \otimes B,
$$

which may be broken up as follows:

$$
\pi^{-1} \pi_{*} A \otimes \pi^{-1} \pi_{*} B \rightarrow A \otimes \pi^{-1} \pi_{*} B \rightarrow A \otimes B .
$$

Therefore the cup product map may be broken up as follows:

$$
\pi_{*} A \otimes \pi_{*} B \rightarrow \pi_{*}\left(A \otimes \pi^{-1} \pi_{*} B\right) \rightarrow \pi_{*}(A \otimes B) .
$$

In our case, this becomes the following sequence of maps:

$$
\pi_{*} u_{!} \mathscr{F} \otimes \pi_{*} u_{*} \mathscr{G} \stackrel{\mu_{1}}{\longrightarrow} \pi_{*}\left(u_{!} \mathscr{F} \otimes \pi^{-1} \pi_{*} u_{*} \mathscr{G}\right) \stackrel{\mu_{2}}{\longrightarrow} \pi_{*}\left(u_{!} \mathscr{F} \otimes u_{*} \mathscr{G}\right) .
$$

Since $\pi$ is a proper map, we know that $\pi_{*} \cong \pi_{\text {! }}$, and hence $\mu_{1}$ is an isomorphism by the projection formula. It remains to show that $\mu_{2}$ is an isomorphism.

The pair of adjoint functors $\left(\pi^{-1}, \pi_{*}\right)$ gives the counit morphism $p^{-1} p_{*} \varphi \rightarrow$ $u^{-1} u_{*} \mathscr{G}$. The key observation is that this map is an isomorphism, because $\mathscr{G}$ is a direct sum of its cohomology sheaves on the affine space $U_{n}$. Now consider the following commutative diagram.

$$
\begin{aligned}
& u_{!} \mathscr{F} \otimes \pi^{-1} \pi_{*} u_{*} G \underset{\text { (proj.) }}{\cong} u_{!}\left(\mathscr{F} \otimes p^{-1} p_{*} \mathscr{G}\right) \\
&\left.\mu_{2}\right|_{(\text {counit })} \cong \downarrow \text { (counit) } \\
& u_{!} \mathscr{F} \otimes u_{*} \mathscr{G} \underset{\text { (proj.) }}{\cong} u_{!}\left(\mathscr{F} \otimes u^{-1} u_{*} \mathscr{G}\right)
\end{aligned}
$$

The map $\mu_{2}$ is obtained by applying the functor $\pi_{*}$ to the left vertical map in (3-4) above. The diagram shows that this map is an isomorphism, and hence $\mu_{2}$ is also an isomorphism.

Lemma 3.2. The cup product map induces an isomorphism

$$
H^{\bullet}\left(u ! u^{-1} L_{1, n}\right) \underset{H(X)}{\otimes} B_{m, n} \cong H_{c}^{\bullet}\left(u^{-1}\left(L_{1, n} \otimes M_{m, n}\right)\right) .
$$

Proof. Using Lemma 3.1 with complexes of sheaves $\mathscr{F}=u^{-1} L_{1, n}$ and $\varphi=u^{-1} L_{2, n}$, we obtain an isomorphism

$$
H^{\bullet}\left(u ! u^{-1} L_{1, n}\right) \underset{H(X)}{\otimes} H^{\bullet}\left(u_{*} u^{-1} L_{2, n}\right) \stackrel{\cong}{\rightarrow} H^{\bullet}\left(u ! u^{-1} L_{1, n} \otimes u_{*} u^{-1} L_{2, n}\right) .
$$

Moreover, $u^{-1} u_{*} u^{-1} L_{2, n} \cong u^{-1} L_{2, n}$. Using this fact and the projection formula,

$$
\begin{aligned}
H^{\bullet}\left(u_{!} u^{-1} L_{1, n} \otimes u_{*} u^{-1} L_{2, n}\right) & \cong H^{\bullet}\left(u_{!}\left(u^{-1} L_{1, n} \otimes u^{-1} u_{*} u^{-1} L_{2, n}\right)\right) \\
& \cong H^{\bullet}\left(u_{!} u^{-1}\left(L_{1, n} \otimes L_{2, n}\right)\right) .
\end{aligned}
$$


All together, we get an isomorphism

$$
H^{\bullet}\left(u ! u^{-1} L_{1, n}\right) \underset{H(X)}{\otimes} H^{\bullet}\left(u_{*} u^{-1} L_{2, n}\right) \stackrel{\cong}{\rightarrow} H^{\bullet}\left(u_{!} u^{-1}\left(L_{1, n} \otimes L_{2, n}\right)\right),
$$

which can be written in our previously introduced notation as

$$
H^{\bullet}\left(u ! u^{-1} L_{1, n}\right) \underset{H(X)}{\otimes} B_{2, n} \cong H^{\bullet}\left(u ! u^{-1}\left(L_{1, n} \otimes M_{2, n}\right)\right) .
$$

Now we can successively tensor the above map over $H(X)$ with the spaces $H^{\bullet}\left(u_{*} u^{-1} L_{i, n}\right)$, with $i$ ranging from 3 to $m$. Each time, we apply Lemma 3.1 for $\mathscr{F}=u^{-1}\left(L_{1, n} \otimes M_{i-1, n}\right)$ and $\mathscr{G}=u^{-1} L_{i, n}$ and use the argument above. Ultimately this construction yields

$$
\begin{aligned}
H^{\bullet}\left(u ! u^{-1} L_{1, n}\right) \underset{H(X)}{\otimes} B_{m, n} & \cong H^{\bullet}\left(u ! u^{-1}\left(L_{1, n} \otimes M_{m-1, n}\right)\right) \underset{H(X)}{\otimes} H^{\bullet}\left(u_{*} u^{-1} L_{m, n}\right) \\
& \cong H^{\bullet}\left(u !\left(u^{-1}\left(L_{1, n} \otimes M_{m, n}\right)\right)\right) \\
& \cong H_{c}^{\bullet}\left(u^{-1}\left(L_{1, n} \otimes M_{m, n}\right)\right) .
\end{aligned}
$$

The next lemma is a refinement of a standard cohomology exact sequence to our particular case.

\section{Lemma 3.3. There is an exact sequence}

$$
H^{\bullet}\left(u ! u^{-1} L_{1, n}\right) \underset{H(X)}{\bigotimes} B_{m, n} \rightarrow H^{\bullet}\left(L_{1, n}\right) \underset{H(X)}{\otimes} A_{m, n} \rightarrow H^{\bullet}\left(v_{*} v^{-1} L_{1, n}\right) \underset{H(X)}{\otimes} A_{m, n} \rightarrow 0 .
$$

Proof. Consider the distinguished triangle (3-1) for the sheaf $L_{1, n}$. Taking cohomology and applying the functor $(-) \underset{H(X)}{\otimes} A_{m, n}$, we obtain the right-exact sequence

$$
H^{\bullet}\left(u ! u^{-1} L_{1, n}\right) \underset{H(X)}{\otimes} A_{m, n} \stackrel{f}{\rightarrow} H^{\bullet}\left(L_{1, n}\right) \underset{H(X)}{\otimes} A_{m, n} \stackrel{g}{\rightarrow} H^{\bullet}\left(v_{*} v^{-1} L_{1, n}\right) \underset{H(X)}{\otimes} A_{m, n} \rightarrow 0 .
$$

Using the distinguished triangles (3-2) for each of the sheaves $L_{j, n}$ for $j \geq 2$, we have surjective morphisms

$$
H^{\bullet}\left(L_{j, n}\right) \rightarrow H^{\bullet}\left(u_{*} u^{-1} L_{j, n}\right) .
$$

Taking the tensor product of all of these along with $H^{\bullet}\left(u_{!} u^{-1} L_{1, n}\right)$, we obtain a surjective morphism

$$
H^{\bullet}\left(u_{!} u^{-1} L_{1, n}\right) \underset{H(X)}{\otimes} A_{m, n} \stackrel{h}{\rightarrow} H^{\bullet}\left(u_{!} u^{-1} L_{1, n}\right) \underset{H(X)}{\otimes} B_{m, n} .
$$

We now show that the map $f$ factors through the map $h$, by showing that $f(\operatorname{ker} h)=0$. Since all boundary maps in the cohomology long exact sequence of the triangles (3-2) vanish, the following set generates $\operatorname{ker} h$ :

$$
\left\{a_{1} \otimes a_{2} \otimes \cdots \otimes a_{n} \mid a_{j} \in H^{\bullet}\left(v_{*} v^{!} L_{j, n}\right) \text { for some } 2 \leq j \leq m\right\} .
$$


Consider any element $a_{1} \otimes a_{2} \otimes \cdots \otimes a_{n} \in \operatorname{ker} h$. Suppose that $a_{j} \in H^{\bullet}\left(v_{*} v^{!} L_{j, n}\right)$. Recall the commutative diagram (3.8a) from [Ginzburg 1991], reproduced below.

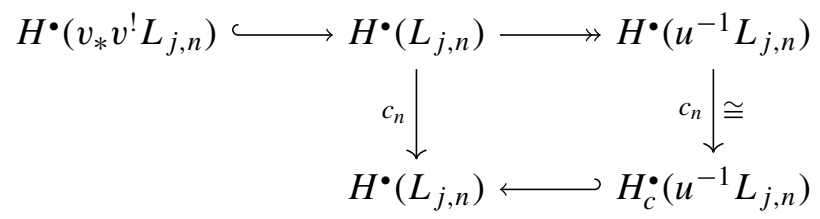

From this diagram it follows that $c_{n} a_{j}=0$, and that $a_{1} \in c_{n} H^{\bullet}\left(L_{1, n}\right)$. Since all tensor products are over $H(X)$, the image of $h\left(a_{1} \otimes \cdots \otimes a_{n}\right)$ under $f$ must be zero. Therefore $f$ factors through $h$, and we obtain the desired short exact sequence.

Finally, we use the induction hypothesis to tackle the right side of the right-exact sequence from the previous lemma.

Lemma 3.4. The cup product map induces an isomorphism

$$
H^{\bullet}\left(v_{*} v^{-1} L_{1, n}\right) \underset{H(X)}{\otimes} A_{m, n} \stackrel{\cong}{\rightrightarrows} H^{\bullet}\left(L_{1, n-1} \otimes M_{m, n-1}\right) .
$$

Proof of lemma. The cup product map on the left hand side is the following composition:

$$
H^{\bullet}\left(v_{*} v^{-1} L_{1, n}\right) \underset{H(X)}{\otimes} A_{m, n} \rightarrow H^{\bullet}\left(v_{*} v^{-1} L_{1, n}\right) \underset{H(X)}{\otimes} H^{\bullet}\left(M_{m, n}\right) \rightarrow H^{\bullet}\left(v_{*} v^{-1} L_{1, n} \otimes M_{m, n}\right),
$$

where the first map is the cup product on the last $(m-1)$ factors, and the second map is the cup product of the first factor with the rest. The projection formula also shows that

$$
H^{\bullet}\left(v_{*} v^{-1} L_{1, n} \otimes M_{m, n}\right) \cong H^{\bullet}\left(v^{-1} L_{1, n} \otimes v^{-1} M_{m, n}\right) \cong H^{\bullet}\left(L_{1, n-1} \otimes M_{m, n-1}\right) .
$$

By induction on $m$, we may assume that the cup product $A_{m, n} \rightarrow H^{\bullet}\left(M_{m, n}\right)$ is an isomorphism, and hence the first map above is an isomorphism. It remains to show that the following map is an isomorphism:

$$
H^{\bullet}\left(v_{*} v^{-1} L_{1, n}\right) \underset{H(X)}{\otimes} H^{\bullet}\left(M_{m, n}\right) \rightarrow H^{\bullet}\left(v_{*} v^{-1} L_{1, n} \otimes M_{m, n}\right)
$$

Since $L_{1, n-1}$ is supported on $X_{n-1}$, the element $c_{n} \in H$ acts on $H^{\bullet}\left(v_{*} L_{1, n-1}\right)$ by zero. Recall from [op. cit.] that the cokernel of $c_{n}$ on $H^{\bullet}\left(M_{m, n}\right)$ is just $H^{\bullet}\left(M_{m, n-1}\right)$. Hence

$$
H^{\bullet}\left(v_{*} v^{-1} L_{1, n}\right) \underset{H(X)}{\otimes} H^{\bullet}\left(M_{m, n}\right) \cong H^{\bullet}\left(L_{1, n-1}\right) \underset{H(X)}{\otimes} H^{\bullet}\left(M_{m, n-1}\right) .
$$

Therefore, the map above can be rewritten as the cup product map

$$
H^{\bullet}\left(L_{1, n-1}\right) \underset{H(X)}{\otimes} H^{\bullet}\left(M_{m, n-1}\right) \rightarrow H^{\bullet}\left(L_{1, n-1} \otimes M_{m, n-1}\right),
$$


which is an isomorphism by the induction hypothesis.

We now apply Saito's theory [1990; 1988] of mixed Hodge modules to obtain another short exact sequence, as follows. Every IC-sheaf has the additional structure of a pure mixed Hodge module, which induces a mixed Hodge structure on tensor products of the $L_{i, n}$.

Lemma 3.5. (i) The cohomology $H^{\bullet}\left(L_{1, n} \otimes M_{m, n}\right)$ is pure.

(ii) There is a short exact sequence

$$
0 \rightarrow H_{c}^{\bullet}\left(u^{-1}\left(L_{1, n} \otimes M_{m, n}\right)\right) \rightarrow H^{\bullet}\left(L_{1, n} \otimes M_{m, n}\right) \rightarrow H^{\bullet}\left(L_{1, n-1} \otimes M_{m, n-1}\right) \rightarrow 0 .
$$

Proof. The proof is by induction on $n$. When $n=0$, we have $X_{-1}=\varnothing$ and $U=X_{0}$. The open inclusion $u$ is the identity map, and the closed inclusion $v$ is the zero map, hence (ii) is clear in the base case.

The set $X_{0}$ consists of a single $T$-fixed point of $X$. Call this point $w$. By Assumption 1.2, there exists a neighborhood $V_{w}$ of $w$ and a one-parameter subgroup $\lambda_{w}: \mathbb{C}^{*} \rightarrow T$ that contracts $V_{w}$ to $w$. Let $i_{w}$ denote the inclusion of $\{w\}$ into the corresponding $V_{w}$. Let $j_{w}$ denote the inclusion of $V_{w}$ into $X$. By applying [Springer 1984, Corollary 1] or [Braden 2003, Lemma 6] to the sheaves $j_{w}^{-1} \mathrm{IC}_{p_{i}}$ for each $i$, we see that

$$
H^{\bullet}\left(V_{w}, j_{w}^{-1} \mathrm{IC}_{p_{i}}\right) \cong H^{\bullet}\left(i_{w}^{-1} j_{w}^{-1} \mathrm{IC}_{p_{i}}\right)=H^{\bullet}\left(L_{i, 0}\right) .
$$

The functor $H^{\bullet}\left(V_{w}, j_{w}^{-1}(-)\right)$ weakly increases weights; on the other hand, the functor $H^{\bullet}\left(i_{w}^{-1} j_{w}^{-1}(-)\right)$ weakly decreases weights. Hence $H^{\bullet}\left(L_{i, 0}\right)$ is pure for each $i$. Taking the tensor product, we see that $H^{\bullet}\left(L_{1,0}\right) \otimes \cdots \otimes H^{\bullet}\left(L_{m, 0}\right)$ is pure. Since $w$ is a single point, we can naturally make the following identification:

$$
H^{\bullet}\left(L_{1,0}\right) \otimes \cdots \otimes H^{\bullet}\left(L_{m, 0}\right) \cong H^{\bullet}\left(L_{1,0} \otimes \cdots \otimes L_{m, 0}\right)=H^{\bullet}\left(L_{1,0} \otimes M_{m, 0}\right) .
$$

Hence $H^{\bullet}\left(L_{1,0} \otimes M_{m, 0}\right)$ is pure, and (i) is proved in the base case. A similar argument has been used in [Ginzburg 1991, Lemma 3.5].

For the induction step, consider the distinguished triangle (3-1) for $L_{1, n}$. Apply the functor $\left(-\otimes L_{2, n} \otimes \cdots \otimes L_{m, n}\right)$, which may be written as $\left(-\otimes M_{m, n}\right)$ in the notation of (3-3). This yields the following distinguished triangle:

$$
u ! u^{-1} L_{1, n} \otimes M_{m, n} \rightarrow L_{1, n} \otimes M_{m, n} \rightarrow v_{*} v^{-1} L_{1, n} \otimes M_{m, n} .
$$

By a repeated application of the projection formula, we may write the first term of this triangle as

$$
u ! u^{-1} L_{1, n} \otimes M_{m, n} \cong u !\left(u^{-1} L_{1, n} \otimes \cdots \otimes u^{-1} L_{m, n}\right)=u ! u^{-1}\left(L_{1, n} \otimes M_{m, n}\right),
$$

and the third term of this triangle as

$$
v_{*} v^{-1} L_{1, n} \otimes M_{m, n} \cong v_{*}\left(v^{-1} L_{1, n} \otimes \cdots \otimes v^{-1} L_{m, n}\right)=v_{*}\left(L_{1, n-1} \otimes M_{m, n-1}\right) .
$$


Taking cohomology, we obtain the following long exact sequence:

$$
\begin{aligned}
\cdots \rightarrow H_{c}^{\bullet}\left(u^{-1}\left(L_{1, n} \otimes M_{m, n}\right)\right) & \rightarrow H^{\bullet}\left(L_{1, n} \otimes M_{m, n}\right) \\
& \rightarrow H^{\bullet}\left(L_{1, n-1} \otimes M_{m, n-1}\right) \rightarrow \cdots .
\end{aligned}
$$

The term $H^{\bullet}\left(L_{1, n-1} \otimes M_{m, n-1}\right)$ is pure by the induction hypothesis.

From Lemma 3.2, we know that

$$
H_{c}^{\bullet}\left(u^{-1}\left(L_{1, n} \otimes M_{m, n}\right)\right) \cong H_{c}^{\bullet}\left(u^{-1} L_{1, n}\right) \underset{H(X)}{\otimes} H^{\bullet}\left(u^{-1} L_{2, n}\right) \underset{H(X)}{\otimes} \cdots \underset{H(X)}{\otimes} H^{\bullet}\left(u^{-1} L_{m, n}\right) .
$$

Recall that $U_{n}$ is the Białynicki-Birula plus cell for the fixed point $w_{n}$. Hence the $\lambda$-action contracts $U_{n}$ to $w_{n}$. By [Springer 1984, Corollary 2], we know that $H_{c}^{\bullet}\left(u^{-1} L_{1, n}\right)$ is isomorphic to the costalk of $u^{-1} L_{1, n}$ at $w_{n}$, which is isomorphic to a shift of the stalk of $\mathrm{IC}_{p_{1}}$ at $w_{n}$. For any $i>1$, we know by [Springer 1984, Corollary 1] that $H^{\bullet}\left(u^{-1} L_{i, n}\right)$ is isomorphic to the stalk of $u^{-1} L_{i, n}$ at $w_{n}$, which is equal to the stalk of $\mathrm{IC}_{p_{i}}$ at $w_{n}$. By using Assumption 1.2 and the argument used earlier in this proof, we know that the stalk of each $\mathrm{IC}_{p_{i}}$ at any $T$-fixed point is pure, and hence the spaces $H_{c}^{\bullet}\left(u^{-1} L_{1, n}\right)$ as well as $H^{\bullet}\left(u^{-1} L_{i, n}\right)$ for $i>1$ are all pure. Therefore the tensor product $H_{c}^{\bullet}\left(u^{-1}\left(L_{1, n} \otimes M_{m, n}\right)\right)$ is pure.

Since the terms on either side of the long exact sequence are pure, the connecting homomorphisms are zero, and hence $H^{\bullet}\left(L_{1, n} \otimes M_{m, n}\right)$ is also pure. This argument completes the induction step, and hence completes the proof.

Putting together the exact sequences from Lemmas 3.3 and 3.5, we obtain the following commutative diagram, where the vertical maps are induced by cup products. In particular, the middle map $b$ is just the map from Proposition 2.1.

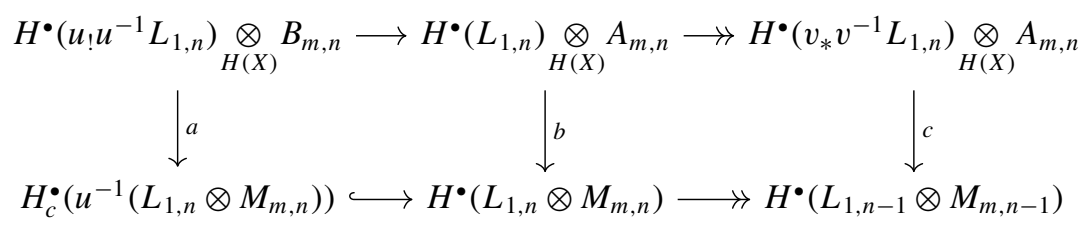

The leftmost map $a$ is an isomorphism by Lemma 3.2. The rightmost map $c$ is an isomorphism by Lemma 3.4. By the snake lemma, the middle map $b$ is an isomorphism as well, and Proposition 2.1 is proved.

\section{Computation of equivariant cohomology}

Consider a smooth complex projective variety $X$ with the same assumptions as in Section 1. The goal of this section is to prove Theorem 1.4.

First, recall some constructions in equivariant cohomology, following [Bernstein and Lunts 1994] and [Goresky et al. 1998]. Fix a universal principal $T$-bundle 
$E T \rightarrow B T$, where $E T$ is the direct limit over $m$ of algebraic approximations $E T_{m}$ and analogously for $B T$ and $B T_{m}$. Consider the following diagram, where the map $p$ is the second projection, and the map $q$ is the quotient by the diagonal $T$-action.

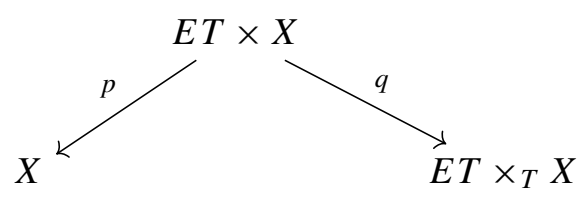

Since each stratum $U_{n}$ is a locally closed $T$-invariant affine subvariety of $X$, the trivial local system on $U_{n}$ gives rise to a canonically defined sheaf $\overline{\mathrm{IC}_{n}}$ on $E T \times_{T} X$ and a canonical isomorphism $\beta: p^{-1} \mathrm{IC}_{n} \cong q^{-1} \overline{\mathrm{IC}_{n}}$ (see, e.g., [Bernstein and Lunts 1994]). The triple $\left(\mathrm{IC}_{n}, \overline{\mathrm{IC}_{n}}, \beta\right)$ is called the equivariant IC sheaf corresponding to $U_{n}$.

Equivariant homology and cohomology. For a variety $Y$ equipped with a $T$-action, the cohomology of $E T \times_{T} Y$ is called the equivariant cohomology of $Y$, and is denoted by $H_{T}^{\cdot}(Y)$. In particular, since $E T \times_{T} \mathrm{pt} \cong B T$, we have $H_{T}^{\cdot}(\mathrm{pt}) \cong H^{\bullet}(B T)$. The space $H_{T}^{\cdot}(Y)$ is a ring under cup product and is also an $H_{T}(X)$-module via pullback under the projection $Y \rightarrow$ pt. For convenience, we will denote $H_{T}^{\bullet}(X)$ by $H_{T}(X)$. In our case, $H_{T}(X)$ is isomorphic to $H^{\bullet}(X) \otimes H^{\bullet}(B T)$ as an $H_{T}(X)$-module (see, e.g., [Goresky et al. 1998, Theorem 14.1]). Similarly, the equivariant cohomology of any $T$-equivariant sheaf on $X$ also carries an $H_{T}(X)$-module structure.

One can define the $T$-equivariant Borel-Moore homology of $X$, denoted $H_{.}^{T}(X)$. Every $T$-equivariant closed subvariety $Y$ of $X$ defines a class $[Y]_{T}$ of degree $2 \operatorname{dim}_{\mathbb{C}} Y$ in $H_{\bullet}^{T}(X)$. If $X$ is smooth, then every class $[Y]_{T}$ has an equivariant Poincaré dual cohomology class in $H_{T}^{\bullet}(X)$. More details can be found in [Graham 2001] and [Brion 2000].

Proof of the equivariant case. Consider an $m$-tuple $\left(p_{1}, \ldots, p_{m}\right)$ of $T$-fixed points of $X$. Then $\mathrm{IC}_{p_{1}}, \ldots, \mathrm{IC}_{p_{m}}$ are the IC sheaves corresponding to $U_{p_{1}}, \ldots, U_{p_{m}}$ respectively. Let $L_{j, n}=i_{n}^{-1} \mathrm{IC}_{p_{j}}$ for each $j$ and $n$.

Proposition 4.1. Under Assumptions 1.1 and 1.2, the cup product maps

$$
H_{T}^{\bullet}\left(L_{1, n}\right) \underset{H_{T}(X)}{\otimes} \cdots \underset{H_{T}(X)}{\otimes} H_{T}^{\cdot}\left(L_{m, n}\right) \rightarrow H_{T}^{\bullet}\left(L_{1, n} \otimes \cdots \otimes L_{m, n}\right)
$$

are isomorphisms for each $n$.

When $X_{n}=X$, we have $L_{j, n}=\mathrm{IC}_{p_{j}}$ for each $j$. Hence this proposition implies Theorem 1.4. To prove the proposition, we first state two general lemmas about $T$-equivariant cohomology of sheaves. 
Lemma 4.2. Consider the fiber bundle $E T \times_{T} X \rightarrow B T$, with fiber $X$. Let $\mathrm{IC}_{w}$ be the (T-equivariant) IC sheaf on the closure of a stratum $X_{w}$, extended by zero to all of $X$. Then the Leray spectral sequence for the computation of $H_{T}^{\cdot}\left(X ; \mathrm{IC}_{w}\right)=$ $H^{\bullet}\left(E T \times_{T} X ; \overline{\mathrm{IC}_{w}}\right)$ collapses at the $E_{2}$ page. Hence $H_{T}^{\bullet}\left(\mathrm{IC}_{w}\right)$ is isomorphic to $H^{\bullet}\left(\mathrm{IC}_{w}\right) \otimes H^{\bullet}(B T)$ as a graded $H^{\bullet}(B T)$-module.

Proof. See [Goresky et al. 1998, Theorem 14.1]. The proof uses the fact that the cohomology of $B T \cong\left(\mathbb{C} P^{\infty}\right)^{\operatorname{dim} T}$ is pure.

Lemma 4.3. Let $Y$ be any $T$-space, and let $\mathscr{F}$ be a T-equivariant sheaf on $Y$ such that the space $H^{\bullet}(Y ; \mathscr{F})$ is pure. Then $H_{T}^{\bullet}(Y ; \mathscr{F})$ is pure as well.

Proof. Recall that $H_{T}^{\bullet}(Y, \mathscr{F})=H^{\bullet}\left(E T \times_{T} X, \overline{\mathscr{F}}\right)$. The result follows from computing the Leray spectral sequence for the fiber bundle $E T \times_{T} Y \rightarrow B T$, and by using that $H^{\bullet}(B T)$ and $H^{\bullet}(Y, \mathscr{F})$ are pure.

We also record some equivariant analogues of results stated in Section 3. First note that the boundary maps in the long exact sequences of $T$-equivariant cohomology for the distinguished triangles (3-1) and (3-2) vanish. The proof is analogous to the nonequivariant case, using Lemma 4.3.

The following lemma is an analogue of Lemma 3.1.

Lemma 4.4. Let $U=X_{n} \backslash X_{n-1}$. Let $\mathscr{F}$ and $\varphi$ be any T-equivariant complexes of sheaves on $U$. Then the cup product map

$$
\cup: H_{T}^{\bullet}\left(u_{!} \mathscr{F}\right) \underset{H^{\bullet}(B T)}{\otimes} H_{T}^{\bullet}\left(u _ { * } ( \mathscr { G } ) \rightarrow H _ { T } ^ { \bullet } \left(u_{!} \mathscr{F} \otimes u_{*}(\mathscr{G})\right.\right.
$$

is an isomorphism. Since $\cup$ factors through the surjection

$$
H_{T}^{\bullet}\left(u_{!} \mathscr{F}\right) \underset{H^{\bullet}(B T)}{\otimes} H_{T}^{\bullet}\left(u_{*}(\mathscr{G}) \rightarrow H_{T}^{\bullet}\left(u_{!} \mathscr{F}\right) \underset{H_{T}(X)}{\otimes} H_{T}^{\bullet}\left(u_{*} \mathscr{G}\right),\right.
$$

the induced cup product

$$
H_{T}^{\bullet}\left(u_{!} \mathscr{F}\right) \underset{H_{T}(X)}{\otimes} H_{T}^{\bullet}\left(u_{*} \mathscr{G}\right) \rightarrow H_{T}^{\bullet}\left(u_{!} \mathscr{F} \otimes u_{*}(\mathscr{G})\right.
$$

is also an isomorphism.

Proof. Consider the fiber bundle $E T \times_{T} X_{n} \rightarrow B T$, with fiber $X_{n}$. The $E_{2}$ pages of the Leray spectral sequences for $u_{!} \mathscr{F}$ and $u_{*} \mathscr{G}$ are as follows:

$$
\begin{aligned}
H^{p}\left(B T, H^{q}\left(u_{!} \mathscr{F}\right)\right) & \Longrightarrow H_{T}^{p+q}\left(u_{!} \mathscr{F}\right), \\
H^{r}\left(B T, H^{s}\left(u_{*} \mathscr{G}\right)\right) & \Longrightarrow H_{T}^{r+s}\left(u_{*} \mathscr{G}\right) .
\end{aligned}
$$

On the $E_{2}$ page, the cup product map can be written as the composition of the following two maps. The first map is the cup product with local coefficients, and 
the second is the fiberwise cup product on the local systems.

$$
\begin{gathered}
H^{p}\left(B T, H^{q}\left(u_{!} \mathscr{F}\right)\right) \underset{H_{\bullet}(B T)}{\otimes} H^{r}\left(B T, H^{s}\left(u_{*}(\mathscr{G})\right) \rightarrow H^{p+r}\left(B T, H^{q}\left(u_{!} \mathscr{F}\right) \otimes H^{s}\left(u_{*} \mathscr{G}\right)\right),\right. \\
H^{p+r}\left(B T, H^{q}(u ! \mathscr{F}) \otimes H^{s}\left(u_{*}(\mathscr{G})\right) \rightarrow H^{p+r}\left(B T, H^{q+s}\left(u_{!} \mathscr{F} \otimes u_{*} \mathscr{G}\right)\right) .\right.
\end{gathered}
$$

Since the local systems $H^{q}\left(u_{!} \mathscr{F}\right)$ and $H^{s}\left(u_{*} \mathscr{G}\right)$ are constant on $B T$, the first map yields isomorphisms

$$
H^{\bullet}\left(B T, H^{q}\left(u_{!} \mathscr{F}\right)\right) \underset{H^{\bullet}(B T)}{\otimes} H^{\bullet}\left(B T, H^{s}\left(u_{*} \mathscr{G}\right)\right) \stackrel{\cong}{\rightrightarrows} H^{\bullet}\left(B T, H^{q}\left(u_{!} \mathscr{F}\right) \otimes H^{s}\left(u_{*} \mathscr{G}\right)\right) .
$$

Finally, we know from Lemma 3.1 that $H^{\bullet}\left(u_{!} \mathscr{F}\right) \otimes H^{\bullet}\left(u_{*} \mathscr{G}\right) \stackrel{\cong}{\rightrightarrows} H^{\bullet}\left(u_{!} \mathscr{F} \otimes u_{*} \mathscr{G}\right)$ via the cup product map. Altogether, the cup product maps on the $E_{2}$ page yield an isomorphism

$$
H^{\bullet}\left(B T, H^{\bullet}\left(u_{!} \mathscr{F}\right)\right) \underset{H^{\bullet}(B T)}{\otimes} H^{\bullet}\left(B T, H^{\bullet}\left(u_{*} \mathscr{G}\right)\right) \stackrel{\cong}{\longrightarrow} H^{\bullet}\left(B T, H^{\bullet}\left(u_{!} \mathscr{F} \otimes u_{*} \mathscr{G}\right)\right) .
$$

The left hand side is a tensor product of two free $H^{\bullet}(B T)$-modules over $H^{\bullet}(B T)$. Hence it converges to

$$
H_{T}^{\bullet}\left(u_{!} \mathscr{F}\right) \underset{H \cdot(B T)}{\otimes} H_{T}^{\bullet}\left(u_{*} \mathscr{G}\right) .
$$

The right hand side converges to $H_{T}^{\bullet}\left(u_{!} \mathscr{F} \otimes u_{*} \mathscr{G}\right)$. Since the $E_{2}$ pages of the left hand side and the right hand side are isomorphic via the cup product map, the following cup product map

$$
H_{T}^{\bullet}\left(u_{!} \mathscr{F}\right) \underset{H^{\bullet}(B T)}{\otimes} H_{T}^{\bullet}\left(u_{*} \mathscr{G}\right) \rightarrow H_{T}^{\bullet}\left(u_{!} \mathscr{F} \otimes u_{*} \mathscr{G}\right)
$$

is an isomorphism.

Let $\widetilde{c_{n}} \in H_{T}(X)$ be the equivariant Poincaré dual of $\left[X_{n}^{-}\right]_{T}$. Each $\widetilde{c}_{n}$ restricts to the class $c_{n}$ under the map $H_{T}(X) \rightarrow H^{\bullet}(X)$, hence the collection $\left\{\widetilde{c}_{n}\right\}$ generates $H_{T}(X)$ over $H^{\bullet}(B T)$.

The following lemma (analogous to [Ginzburg 1991, (3.8a)]) describes the action of $\widetilde{c_{n}}$ on the equivariant cohomology of the sheaves $L_{j, n}$ on $X$.

Lemma 4.5. For every $j$, the action of $\widetilde{c}_{n}$ on $H_{T}^{\cdot}\left(L_{j, n}\right)$ fits into the following commutative diagram:

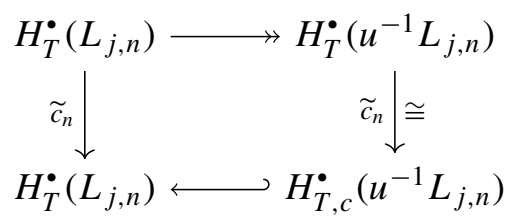


Proof. Recall that the intersection of $X_{n}$ and $X_{n}^{-}$lies away from $X_{n-1}$. Hence $\widetilde{c}_{n}$ restricts to zero on $X_{n-1}$, and cup product by $\widetilde{c}_{n}$ annihilates the cohomology of any sheaf supported on $X_{n-1}$. The kernel of $H_{T}^{\cdot}\left(L_{j, n}\right) \rightarrow H_{T}^{\bullet}\left(u^{-1} L_{j, n}\right)$ and the cokernel of $H_{T, c}^{\bullet}\left(u^{-1} L_{j, n}\right) \rightarrow H_{T}^{\bullet}\left(L_{j, n}\right)$ are both supported on $X_{n-1}$. So the map of multiplication by $\widetilde{c_{n}}$ from $H_{T}^{\bullet}\left(X_{n}\right)$ to $H_{T}^{\bullet}\left(X_{n}\right)$ factors as follows.

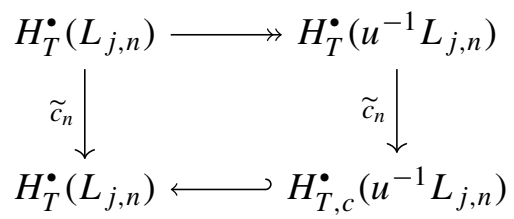

It remains to show that the vertical map on the right is an isomorphism. Since $X_{n}$ and $X_{n}^{-}$intersect transversally in the single point $w_{n}$, the restriction of $\widetilde{c}_{n}$ to $X_{n}$ is the image in $H_{T}^{\cdot}\left(X_{n}\right)$ of a generator of the local cohomology group $H_{T}^{\cdot}\left(X_{n}, X_{n} \backslash\left\{w_{n}\right\}\right)$.

Since $w_{n} \in U_{n}$, we have $H_{T}^{\bullet}\left(X_{n}, X_{n} \backslash\left\{w_{n}\right\}\right) \cong H_{T}^{\bullet}\left(U_{n}, U_{n} \backslash\left\{w_{n}\right\}\right)$ by excision. But $U_{n}$ is an affine space that is $T$-equivariantly contractible to $w_{n}$, and hence $H_{T}^{\bullet}\left(U_{n}, U_{n} \backslash\left\{w_{n}\right\}\right) \cong H_{T, c}^{\bullet}\left(U_{n}\right)$. This shows that multiplication by $\widetilde{c}_{n}$ maps $H_{T}^{\bullet}\left(U_{n}\right)$ isomorphically to $H_{T, c}^{\cdot}\left(U_{n}\right)$.

Since $u^{-1} L_{j, n}$ is $T$-equivariant, the above argument applies to the cohomology of $u^{-1} L_{j, n}$ as well. This means that $\widetilde{c_{n}}$ maps $H_{T}^{\cdot}\left(u^{-1} L_{j, n}\right)$ isomorphically to $H_{T, c}^{\bullet}\left(u^{-1} L_{j, n}\right)$, and the proof is complete.

Once again, let $M_{m, n}$ denote the sheaf $L_{2, n} \otimes \cdots \otimes L_{m, n}$. For brevity, we set up the following additional notation.

$$
\begin{aligned}
& \bar{A}_{m, n}=H_{T}^{\bullet}\left(L_{2, n}\right) \underset{H_{T}(X)}{\otimes} \cdots \underset{H_{T}(X)}{\otimes} H_{T}^{\bullet}\left(L_{m, n}\right), \\
& \bar{B}_{m, n}=H_{T}^{\bullet}\left(u_{*} u^{-1} L_{2, n}\right) \underset{H_{T}(X)}{\otimes} \cdots \underset{H_{T}(X)}{\otimes} H_{T}^{\bullet}\left(u_{*} u^{-1} L_{m, n}\right) .
\end{aligned}
$$

The following two lemmas are analogues of Lemmas 3.3 and 3.5, respectively.

Lemma 4.6. There is an exact sequence

$$
H_{T}^{\bullet}\left(u_{!} u^{-1} L_{1, n}\right) \underset{H_{T}(X)}{\otimes} \bar{B}_{m, n} \rightarrow H_{T}^{\bullet}\left(L_{1, n}\right) \underset{H_{T}(X)}{\otimes} \bar{A}_{m, n} \rightarrow H_{T}^{\bullet}\left(v_{*} v^{-1} L_{1, n}\right) \underset{H_{T}(X)}{\otimes} \bar{A}_{m, n} \rightarrow 0 .
$$

Proof. The proof is analogous to the proof of Lemma 3.3. We use the fact that $H_{T}^{\cdot}(X) \cong H^{\bullet}(X) \otimes H^{\bullet}(B T)$ and use Lemma 4.5 as a substitute for the commutative diagram (3.8a) in [Ginzburg 1991].

Lemma 4.7. (i) The cohomology $H_{T}^{\bullet}\left(L_{1, n} \otimes M_{m, n}\right)$ is pure.

(ii) There is a short exact sequence

$0 \rightarrow H_{T, c}^{\bullet}\left(u^{-1}\left(L_{1, n} \otimes M_{m, n}\right)\right) \rightarrow H_{T}^{\bullet}\left(L_{1, n} \otimes M_{m, n}\right) \rightarrow H_{T}^{\bullet}\left(L_{1, n-1} \otimes M_{m, n-1}\right) \rightarrow 0$. 
Proof. The proofs are analogous to the proofs of their counterparts from Section 3, using the observation of Lemma 4.3 and the fact that $H^{\bullet}(B T)$ is pure.

We now complete the proof of Theorem 1.4.

Proof of Theorem 1.4. We obtain the following commutative diagram from the exact sequences of Lemmas 4.6 and 4.7.

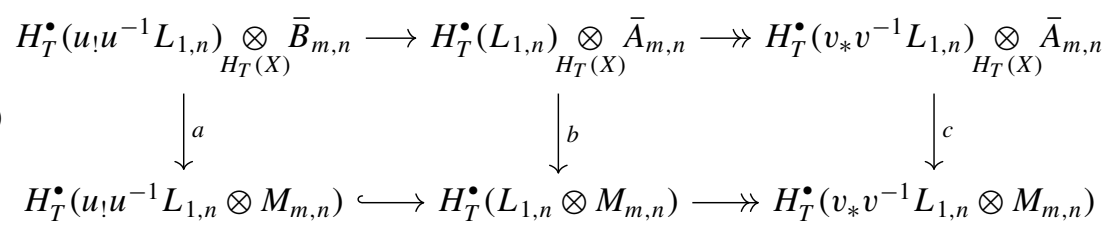

First observe that the action of $H_{T}(X)$ on $H_{T}^{\bullet}\left(u ! u^{-1} L_{1, n}\right)$ and on $\bar{B}_{m, n}$ factors through the map $H_{T}(X) \rightarrow H_{T}^{\bullet}(U) \cong H^{\bullet}(B T)$, so

$$
H_{T}^{\bullet}\left(u ! u^{-1} L_{1, n}\right) \underset{H_{T}(X)}{\otimes} \bar{B}_{m, n} \cong H_{T}^{\bullet}\left(u ! u^{-1} L_{1, n}\right) \underset{H^{\bullet}(B T)}{\otimes} \bar{B}_{m, n} .
$$

We prove by induction on $m$ that the map $a$ is an isomorphism. As in the proof of Lemma 3.2, the case of $m=2$ is proved by Lemma 4.4, and the general case is proved by iterating the argument. An argument similar to the proof of Lemma 3.4 proves that the map $c$ is an isomorphism.

Hence by the snake lemma, the middle map $b$ is an isomorphism as well. Consequently, we obtain the following isomorphisms for every $n$ :

$$
H_{T}^{\bullet}\left(L_{1, n}\right) \underset{H_{T}(X)}{\otimes} \cdots \underset{H_{T}(X)}{\otimes} H_{T}^{\bullet}\left(L_{m, n}\right) \rightarrow H_{T}^{\bullet}\left(L_{1, n} \otimes \cdots \otimes L_{m, n}\right) .
$$

In particular when $X_{n}=X$, we see that the cup product map

$$
H_{T}^{\cdot}\left(\mathrm{IC}_{p_{1}}\right) \underset{H_{T}(X)}{\otimes} \cdots \underset{H_{T}(X)}{\otimes} H_{T}^{\cdot}\left(\mathrm{IC}_{p_{m}}\right) \rightarrow H_{T}^{\bullet}\left(\mathrm{IC}_{p_{1}} \otimes \cdots \otimes \mathrm{IC}_{p_{m}}\right)
$$

is an isomorphism.

Acknowledgments. I am extremely grateful to Victor Ginzburg for suggesting the problem, and for his valuable advice and continued guidance throughout the project. I am grateful to the anonymous referee for providing detailed feedback and several improvements. I thank Quoc Ho for many inspiring mathematical conversations.

\section{References}

[Achar and Rider 2014] P. N. Achar and L. Rider, "Parity sheaves on the affine Grassmannian and the Mirković-Vilonen conjecture”, preprint, 2014. arXiv 1305.1684

[Audin 2004] M. Audin, Torus actions on symplectic manifolds, 2nd ed., Progress in Mathematics 93, Birkhäuser, Basel, 2004. MR 2005k:53158 Zbl 1062.57040 
[Bernstein and Lunts 1994] J. Bernstein and V. Lunts, Equivariant sheaves and functors, Lecture Notes in Mathematics 1578, Springer, Berlin, 1994. MR 95k:55012 Zbl 0808.14038

[Białynicki-Birula 1973] A. Białynicki-Birula, "Some theorems on actions of algebraic groups", Ann. of Math. (2) 98 (1973), 480-497. MR 51 \#3186 Zbl 0275.14007

[Braden 2003] T. Braden, "Hyperbolic localization of intersection cohomology", Transform. Groups 8:3 (2003), 209-216. MR 2004f:14037 Zbl 1026.14005

[Brion 2000] M. Brion, "Poincaré duality and equivariant (co)homology", Michigan Math. J. 48 (2000), 77-92. MR 2001m:14032 Zbl 1077.14523

[Chriss and Ginzburg 1997] N. Chriss and V. Ginzburg, Representation theory and complex geometry, Birkhäuser, Boston, 1997. Reprinted Springer, 2010. MR 98i:22021 Zbl 0879.22001

[Ginzburg 1991] V. Ginsburg, "Perverse sheaves and $\mathbb{C}^{*}$-actions", J. Amer. Math. Soc. 4:3 (1991), 483-490. MR 92d:14013 Zbl 0760.14008

[Goresky et al. 1998] M. Goresky, R. Kottwitz, and R. MacPherson, "Equivariant cohomology, Koszul duality, and the localization theorem”, Invent. Math. 131:1 (1998), 25-83. MR 99c:55009 Zbl 0897.22009

[Graham 2001] W. Graham, "Positivity in equivariant Schubert calculus", Duke Math. J. 109:3 (2001), 599-614. MR 2002h:14083 Zbl 1069.14055

[Juteau et al. 2014] D. Juteau, C. Mautner, and G. Williamson, "Parity sheaves", J. Amer. Math. Soc. 27:4 (2014), 1169-1212. MR 3230821 Zbl 06355534

[Kambayashi 1966] T. Kambayashi, "Projective representation of algebraic linear groups of transformations", Amer. J. Math. 88 (1966), 199-205. MR 34 \#5826 Zbl 0141.18303

[Saito 1988] M. Saito, "Modules de Hodge polarisables", Publ. Res. Inst. Math. Sci. 24:6 (1988), 849-995. MR 90k:32038 Zbl 0691.14007

[Saito 1990] M. Saito, "Mixed Hodge modules", Publ. Res. Inst. Math. Sci. 26:2 (1990), 221-333. MR 91m:14014 Zbl 0727.14004

[Soergel 1990] W. Soergel, "Kategorie O, perverse Garben und Moduln über den Koinvarianten zur Weylgruppe”, J. Amer. Math. Soc. 3:2 (1990), 421-445. MR 91e:17007 Zbl 0747.17008

[Springer 1984] T. A. Springer, "A purity result for fixed point varieties in flag manifolds", J. Fac. Sci. Univ. Tokyo Sect. IA Math. 31:2 (1984), 271-282. MR 86c:14034 Zbl 0581.20048

[Sumihiro 1974] H. Sumihiro, "Equivariant completion”, J. Math. Kyoto Univ. 14:1 (1974), 1-28. MR 49 \#2732 Zbl 0277.14008

Received July 8, 2014. Revised December 12, 2014.

\author{
Asilata BAPAT \\ DEPARTMENT OF MATHEMATICS \\ THE UNIVERSITY OF CHICAGO \\ 5734 S UNIVERSITY AVENUE \\ ChicAgo, IL 60637 \\ UNITED STATES \\ asilata@math.uchicago.edu
}




\title{
PACIFIC JOURNAL OF MATHEMATICS
}

\author{
msp.org/pjm
}

Founded in 1951 by E. F. Beckenbach (1906-1982) and F. Wolf (1904-1989)

\section{EDITORS}

Don Blasius (Managing Editor)

Department of Mathematics

University of California

Los Angeles, CA 90095-1555

blasius@math.ucla.edu

\author{
Paul Balmer \\ Department of Mathematics \\ University of California \\ Los Angeles, CA 90095-1555 \\ balmer@math.ucla.edu \\ Robert Finn \\ Department of Mathematics \\ Stanford University \\ Stanford, CA 94305-2125 \\ finn@math.stanford.edu \\ Sorin Popa \\ Department of Mathematics \\ University of California \\ Los Angeles, CA 90095-1555 \\ popa@math.ucla.edu
}

\author{
Vyjayanthi Chari \\ Department of Mathematics \\ University of California \\ Riverside, CA 92521-0135 \\ chari@math.ucr.edu \\ Kefeng Liu \\ Department of Mathematics \\ University of California \\ Los Angeles, CA 90095-1555 \\ liu@math.ucla.edu \\ Jie Qing \\ Department of Mathematics \\ University of California \\ Santa Cruz, CA 95064 \\ qing@ cats.ucsc.edu
}

\section{PRODUCTION}

Silvio Levy, Scientific Editor, production@msp.org

\section{SUPPORTING INSTITUTIONS}

ACADEMIA SINICA, TAIPEI

CALIFORNIA INST. OF TECHNOLOGY

INST. DE MATEMÁTICA PURA E APLICADA

KEIO UNIVERSITY

MATH. SCIENCES RESEARCH INSTITUTE

NEW MEXICO STATE UNIV.

OREGON STATE UNIV.

\author{
STANFORD UNIVERSITY \\ UNIV. OF BRITISH COLUMBIA \\ UNIV. OF CALIFORNIA, BERKELEY \\ UNIV. OF CALIFORNIA, DAVIS \\ UNIV. OF CALIFORNIA, LOS ANGELES \\ UNIV. OF CALIFORNIA, RIVERSIDE \\ UNIV. OF CALIFORNIA, SAN DIEGO \\ UNIV. OF CALIF., SANTA BARBARA
}

\author{
Daryl Cooper \\ Department of Mathematics \\ University of California \\ Santa Barbara, CA 93106-3080 \\ cooper@math.ucsb.edu \\ Jiang-Hua Lu \\ Department of Mathematics \\ The University of Hong Kong \\ Pokfulam Rd., Hong Kong \\ jhlu@maths.hku.hk \\ Paul Yang \\ Department of Mathematics \\ Princeton University \\ Princeton NJ 08544-1000 \\ yang@math.princeton.edu
}

These supporting institutions contribute to the cost of publication of this Journal, but they are not owners or publishers and have no responsibility for its contents or policies.

See inside back cover or msp.org/pjm for submission instructions.

The subscription price for 2015 is US \$420/year for the electronic version, and \$570/year for print and electronic.

Subscriptions, requests for back issues and changes of subscribers address should be sent to Pacific Journal of Mathematics, P.O. Box 4163, Berkeley, CA 94704-0163, U.S.A. The Pacific Journal of Mathematics is indexed by Mathematical Reviews, Zentralblatt MATH, PASCAL CNRS Index, Referativnyi Zhurnal, Current Mathematical Publications and Web of Knowledge (Science Citation Index).

The Pacific Journal of Mathematics (ISSN 0030-8730) at the University of California, c/o Department of Mathematics, 798 Evans Hall \#3840, Berkeley, CA 94720-3840, is published twelve times a year. Periodical rate postage paid at Berkeley, CA 94704, and additional mailing offices. POSTMASTER: send address changes to Pacific Journal of Mathematics, P.O. Box 4163, Berkeley, CA 94704-0163.

PJM peer review and production are managed by EditFLOW ${ }^{\circledR}$ from Mathematical Sciences Publishers.

\section{PUBLISHED BY}

\section{mathematical sciences publishers \\ nonprofit scientific publishing}

http://msp.org/

(C) 2015 Mathematical Sciences Publishers 


\section{PACIFIC JOURNAL OF MATHEMATICS}

Volume $276 \quad$ No. $1 \quad$ July 2015

On the degree of certain local $L$-functions

U. K. ANANDAVARdhanan and AmiYa Kumar MondaL

Torus actions and tensor products of intersection cohomology

ASILATA BAPAT

Cyclicity in Dirichlet-type spaces and extremal polynomials II: functions on the bidisk

Catherine Bénéteau, Alberto A. Condori, Constanze Liaw,

DANIEl SECo and Alan A. SOlA

Compactness results for sequences of approximate biharmonic maps

CHRISTINE BREINER and TOBIAS LAMM

Criteria for vanishing of Tor over complete intersections

Olgur Celikbas, Srikanth B. Iyengar, Greg PiePMeyer and

ROGER WIEGAND

Convex solutions to the power-of-mean curvature flow

\section{SHIBING CHEN}

Constructions of periodic minimal surfaces and minimal annuli in $\mathrm{Sol}_{3}$

CHRISTOPHE DESMONTS

Quasi-exceptional domains

ALEXANDRE EREMENKO and ERIK LUNDBERG

Endoscopic transfer for unitary groups and holomorphy of Asai $L$-functions

NEVEN GRBAC and FREYDOON SHAHIDI

Quasiconformal harmonic mappings between Dini-smooth Jordan domains

DAVID KALAJ

Semisimple super Tannakian categories with a small tensor generator

THOMAS KRÄMER and RAINER WEISSAUER

On maximal Lindenstrauss spaces

PETR PETRÁČEK and JiŘí SPURNÝ 